\title{
Emergency Medical Service (EMS) Utilization by Syrian Refugees Residing in Ankara, Turkey
}

\author{
Ali Osman Altıner; ${ }^{1}$ SıdıkaTekeli Yeşil, MPH, $\mathrm{PhD}^{2}$
}

1. 112 Emergency Medical Service, Ankara, Turkey

2. Swiss Tropical and Public Health Institute, Department of Medicine, Clinical

Research Unit, Basel, Switzerland

\section{Correspondence:}

Sıdıka Tekeli Yeşil, MPH, PhD

Swiss Tropical and Public Health Institute

Department of Medicine, Clinical

Research Unit

P.O. Box 4002 Basel, Switzerland

E-mail: sidika.tekeli-yesil@swisstph.ch

Conflicts of interest: none

Keywords: Ankara; emergency; Emergency

Medical Services; Syrian refugees; Turkey

Abbreviations:

CHC: Community Health Center

EMS: Emergency Medical Services

FHC: Family Health Center

MHC: Migrant Health Center

Received: December 31, 2016

Revised: August 10, 2017

Accepted: August 13, 2017

Online publication: February 19, 2018

doi:10.1017/S1049023X18000134

\begin{abstract}
Introduction: Many Syrians have left their country and migrated to other countries since March 2011, due to the civil war. As of March 2016, a total of 2,747,946 Syrian refugees had immigrated to Turkey. Some Syrian refugees have been living in camps, while 2,475,134 have been living in metropolitan areas, such as Ankara.

Study Objective: This study investigated Emergency Medical Service (EMS) utilization among Syrian refugees residing in Ankara.

Methods: This study was a descriptive, cross-sectional database analysis using data obtained from the Department of EMS of the Ankara Provincial Health Directorate.

Conclusion: Five stations in the Altındağ region of Ankara responded to $42 \%$ of all calls from Syrian refugees. Prehospital EMS in Ankara have been used mostly by Syrian refugees younger than 18-years-old. Study findings also suggest that medical staff in regions where Syrian refugees are likely to be treated should be supported and provided with the ability to overcome language barriers and cultural differences.
\end{abstract}

Altıner AO, Tekeli Yeşil S. Emergency Medical Service (EMS) utilization by Syrian refugees residing in Ankara, Turkey. Prehosp Disaster Med. 2018;33(2):160-164.

\section{Introduction}

Since the beginning of the conflict in Syria in 2011, millions of Syrians have fled their homes and migrated to neighboring, or more distant countries, with Lebanon, Jordan, Turkey, and North Africa having the greatest influx of Syrian migrants. ${ }^{1}$ Turkey, the largest host country among those mentioned ${ }^{2}$ (Figure 1), has made it clear that it will not close its borders to those escaping the war and that it will welcome them in line with its "open door" policy and provide "temporary protection." In this paper, the term "refugee" is used to describe Syrians under temporary protection of Turkey.

The number of Syrian refugees in Turkey was 14,237 in 2012, 224,655 in 2013, $1,504,112$ in 2014 , and 2,503,549 in 2015; by 2016, the Syrian refugee population had reached a total of 2,758,409. Of them, 272,812 were hosted in camps and 2,475,135 were living outside of camps. ${ }^{3}$ Most Syrians living outside of camps were residing in urban areas for job opportunities, with Ankara one of these cities.

Turkey enabled Syrian refugees to benefit from all of the health care services at all levels provided for its own citizens. Health services for Syrian refugees inside and outside of camps are provided free of charge. These services can be categorized into four groups:

1. Prehospital and EMS, as well as routine emergency health services provided in Turkey: prehospital and EMS are also provided to wounded Syrians in regions close to the Syrian-Turkish border;

2. Health care services at refugee camps: all camps have Community Health Centers (CHCs), which provide primary health care services and outpatient services in some specialties; some camps also have hospitals, depending on their population;

3. Family and migrant health centers: in Turkey, primary health care services are delivered in CHCs and Family Health Centers (FHCs); Syrian refugees have been using these centers also. Following the refugee influx, Turkey formed Migrant Health Centers (MHCs) attached to the FHCs and have been assigning Arabic-speaking personal to those centers (the establishment of MHCs did not limit access to health services from other centers or institutions); and

4. Secondary and tertiary health care services: Syrians benefit from all health services provided by secondary- or tertiary-level hospitals. 


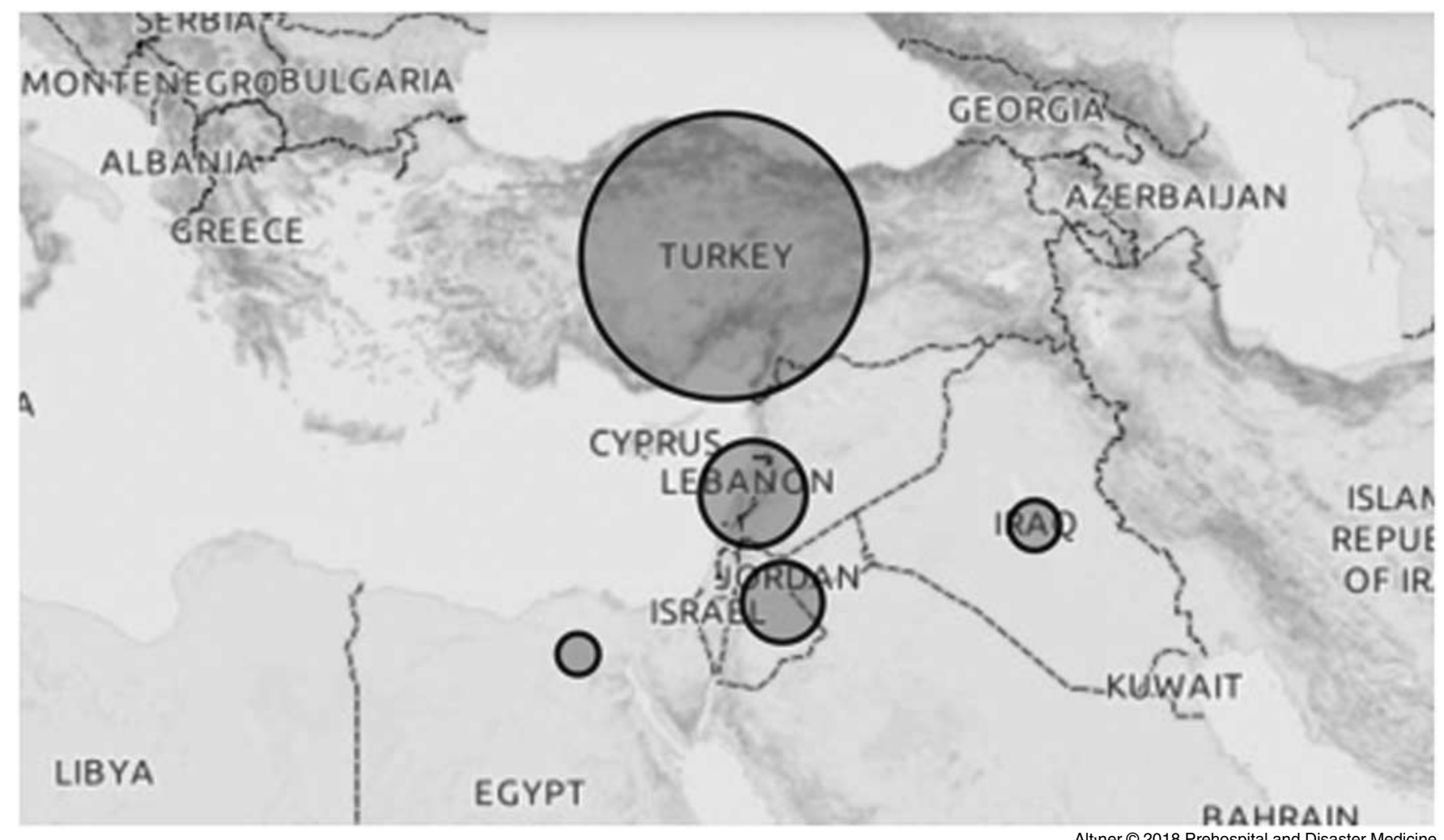

Figure 1. Distribution of Syrian Migrants Hosted in Countries Neighboring Syria. ${ }^{2}$

Altıner $\odot 2018$ Prehospital and Disaster Medicine

According to the statement of the Ex-Directorate General of the Emergency Health Services of Ministry of Health at the beginning of 2015, a total of 3,164,164 refugees benefited from outpatient services at camps; 3,366,804 benefited at non-camp settings; and 576,748 were referred to hospitals from camps. In total, 249,230 Syrians benefited from inpatient treatment, 199,165 were operated on, and 40,823 delivered babies as of the beginning of $2015 ;^{4}$ these numbers nearly doubled by the end of 2015. Extensive use of the Turkish health care system in caring for the health needs of the Syrian refugees placed a considerable burden on the system. Unfortunately, limited literature is available regarding this subject at the national and international levels. Doocy, et al focused on utilization and costs for health care services provided to Syrian child refugees in Jordan. ${ }^{5}$ Ay, et al studied accessibility to health care services among non-camp Syrian refuges in Jordan. ${ }^{6}$ According to a World Vision (Monrovia, California USA) report, local people in Lebanon had difficulty accessing ambulance services, as existing public resources were shared with Syrian refugees. ${ }^{7}$ However, no national study or assessment report regarding utilization of prehospital emergency services was found in this study.

Emergency Medical Services have a significant impact in health care systems. ${ }^{8,9}$ The EMS provides uninterrupted, 24-hour prehospital EMS in Turkey. The number of Syrian refugees who benefit from this prehospital EMS has increased, along with the Turkish population. This study was conducted to determine EMS utilization among Syrian refugees residing in Ankara in an attempt to facilitate future EMS planning and arrangements.

\section{Material and Methods}

This was a descriptive, cross-sectional study using data obtained from the Department of EMS of the Ankara Provincial Health
Directorate database. Data are entered into the database by trained personal of the Health Directorate and double checked for accuracy. Use of the database for this study was approved by the Ankara Provincial Health Directorate, as well as an ethical clearance from the Hacettepe University's Ethical Committee (Ankara, Turkey). Records from January 1, 2013 through June 1, 2015 were analyzed. Calls made by Syrians and responded to by ambulance were included in the study. People under the temporary protection in Turkey receive an ID number coded differently than Turkish citizens, and this ID number system was used to determine when a Syrian refugee accessed the EMS system. All entries to the EMS database are done using the ID numbers. Date, call time, name, and code of the ambulance station, as well as gender, age, reason for the call, pre-diagnosis, and receiving hospital, were the variables obtained and entered into study data forms. Among these variables: (i) "Reason for call," defined as why the ambulance was called, was sub-grouped in the database as medical, traffic accident, work accident, other accident, fire, suicide, protocol, health precaution, and other; (ii) "Pre-diagnosis" was defined as the diagnosis by the ambulance team leader at the scene; (iii) "Results" was defined as how the emergency case ended and was further subdivided into transportation to the hospital, refusal of transportation, transportation with another vehicle, and exit by EMS and left at the scene; and (iv) "Receiving hospital" was defined as the hospital where the patient was transferred. According to information obtained from the Ankara Health Directorate database, 2,943 Syrian cases were reported during the aforementioned period. However, some of the calls were grouped as "repeated calls" as they were taken from the same stations at the same time and from the same age and gender groups. Entering more than one diagnosis for the same case caused the calls to be reported as different 


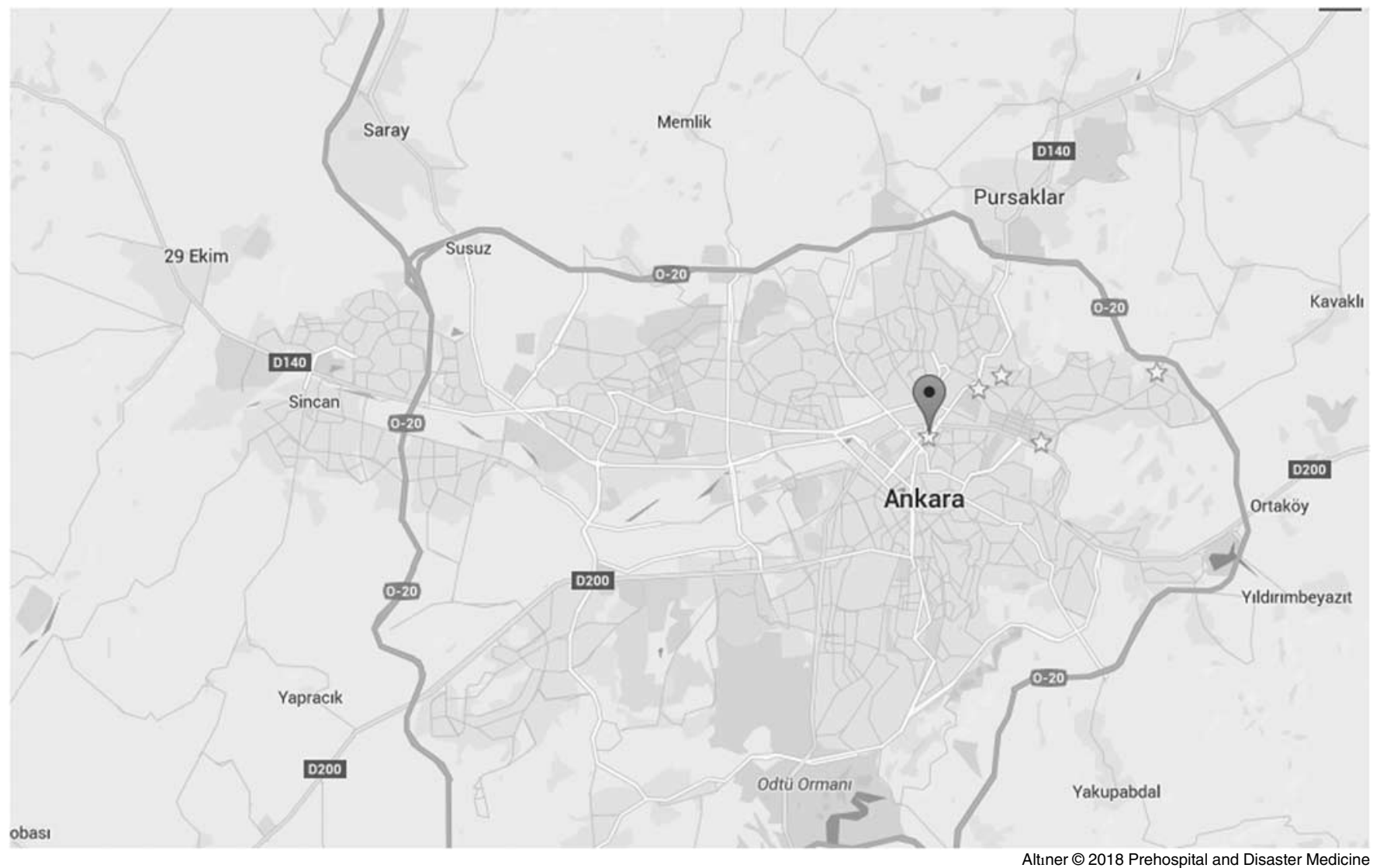

Figure 2. Locations of First Five Ambulance Stations (marked by star).

cases. In such cases, the first pre-diagnosis was taken into consideration, and after adjustment for duplicate cases, 2,270 calls were entered into the study.

The distributions of all variables were analyzed and cross-tables were created. Age was categorized in six groups as: 0 to $<1,1$ to $<5,5-17,18-45,46-60$, and $>60$. The chi-square test was used to test the significance of the cross-tables. The data were analyzed with IBM SPSS Statistics 22 software (IBM Corp; Armonk, New York USA).

\section{Results}

A total of 126 of 133 ambulance stations in Ankara responded to Syrian refugee calls from January 1, 2013 through June 1, 2015. Five stations responded to $42.0 \%$ of all Syrian refugee calls: Siteler $(n=296)$; Hüseyin Gazi $(n=236)$; Gülpınar $(n=215)$; Hasköy $(n=140)$; and Dışkapı $(n=67)$. These stations were in close proximity to each other and were located in the Altındağ region of Ankara (Figure 2).

The use of the ambulance service by Syrians rose over the years from 2013 to 2015: 204 of 319,160 calls responded to by ambulance were made by Syrians in 2013 (0.06\%; 95\% CI, 0.0550.073). In 2014, 1,128 of 332,370 calls were from Syrian refugees (0.33\%; 95\% CI, 0.32-0.35), and 938 of 179,243 of ambulance calls were from Syrians in the first five months of 2015 (0.52\%; 95\% CI, 0.49-0.55; Figure 3).

An equal gender distribution of calls was observed according to the callers (female: $51 \%, n=1,148,95 \% \mathrm{CI}, 48-54$; and male: $49 \%, \mathrm{n}=1122,95 \% \mathrm{CI}, 47-52)$.

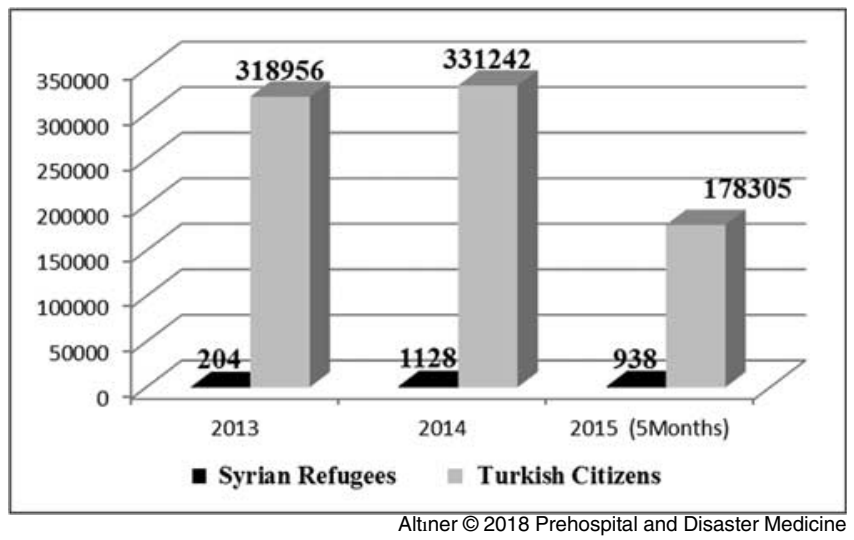

Figure 3. Distribution of Calls Responded with Ambulance in Ankara per Year and the User.

When the calls were analyzed according to case outcome, 1,642 cases were transported to hospitals, 250 cases refused transport, 223 were transported between hospitals, 64 were treated at the scene, 49 were transported to home, 19 were transported in another vehicle, and 11 were transported for medical examination. Eleven cases were left at the scene. One of the ambulance response calls did not have a recorded result.

Figure 4 shows the receiving hospital type according to funding. In total, 1,793 Syrians were transported to public hospitals, 49 to university hospitals, and 20 were transported to private hospitals. The remaining 402 Syrians were not transported to 


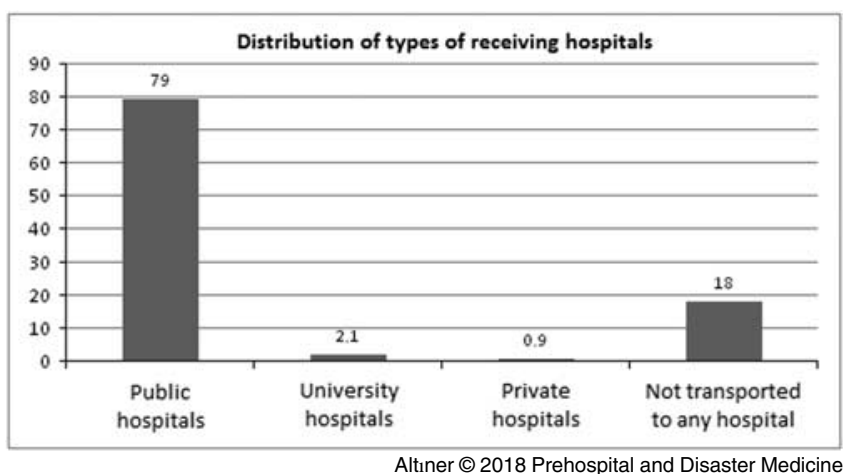

Figure 4. Analysis of Receiving Hospital Types (\%).

any hospital. Almost all transported Syrians (96.3\%) were received by public hospitals.

The first five receiving public hospitals were: Dıskapı Yıldırım Beyazıt Training and Research Hospital $(n=480)$; Ankara Training and Research Hospital $(n=380)$; Dr. Sami Ulus Maternity and Children's Health and Disease Training and Research Hospital $(\mathrm{n}=159)$; Dr. Zekai Tahir Burak Women's Healthcare Training and Research Hospital $(\mathrm{n}=139)$; and Numune Training and Research Hospital $(\mathrm{n}=136)$. These hospitals received $69.4 \%$ of transported Syrians. When the transports were analyzed according to patient gender, Ankara Training and Research Hospital received 200 female patients. Dışkapı Yıldırım Beyazit Training and Research Hospital, Dr. Zekai Tahir Burak Women's Healthcare Training and Research Hospital, Dr. Sami Ulus Maternity and Children's Health and Disease Training and Research Hospital, and Etlik Training and Research Hospital each received 180, 135, 101, and 75 female Syrians, respectively.

Calls made by Syrians and responded to by ambulance had a diverse age distribution (Mean: 25; Medium: 23; Min: 0; Max: 104; SD: 21). Most of the cases were in the 18-45 years age group (41.0\%; $\mathrm{n}=932$ ). The distributions of the other groups were: $0-1$ year age group $(16.0 \% ; n=363) ; 5-17$ years age group (14.3\%; $\mathrm{n}=325) ; 46-60$ years age group $(8.5 \% ; \mathrm{n}=193) ; \geq 60$ years age group $(6.3 \% ; n=144)$; and $1-5$ years age group $(5.9 \% ; n=133)$. Ambulances were primarily called for patients $<18$-years-old $(36.1 \% ; \mathrm{n}=821)$; the age group of 180 patients was not recorded (Table 1).

The first three reasons for calls were medical $(n=1,609)$, health precaution $(n=224)$, and traffic accident $(n=173)$. The detailed distribution for the reasons for the calls is given in Table 2.

Further analysis was performed by categorizing the reasons for the calls into accident and medical groups. Suicide, injury, other accidents, work accidents, traffic accidents, and fire were listed under the accident group. Health precaution, medical, and other reasons were categorized as medical.

The reasons for the calls were analyzed according to gender. Most ambulance calls were for medical reasons and 926 of the cases were females, whereas 683 were males. The frequency of calls for medical reasons was significantly higher among females than that in males $\left(\mathrm{X}^{2}=138.057 ; \mathrm{P}<.001\right)$.

Table 3 lists the distribution of ambulance calls by Syrians according to time interval. There were 337 calls made during the 00:00-12:00 hour interval, 416 calls during the 06:00-12:00 interval, 770 calls during the 12:00-18:00 interval, and 747 calls during 18:00-00:00 interval. The busiest time interval was between noon and midnight.

\begin{tabular}{|l|c|c|c|}
\hline Age Group & Number (n) & Percent (\%) & $\mathbf{9 5 \% ~ C l}$ \\
\hline $0-1$ & 363 & 16.0 & $14.4-17.7$ \\
\hline $1-5$ & 133 & 5.9 & $4.9-6.9$ \\
\hline $5-18$ & 325 & 14.3 & $12.8-15.9$ \\
\hline $18-45$ & 932 & 41.0 & $38.4-43.8$ \\
\hline $45-60$ & 193 & 8.5 & $7.4-9.8$ \\
\hline $60+$ & 144 & 6.3 & $5.4-7.5$ \\
\hline Not Recorded & 180 & 8.0 & - \\
\hline
\end{tabular}

Table 1. Distribution of Calls According to Patient's Age

\begin{tabular}{|l|c|}
\hline Medical & 1609 \\
\hline Health Precaution & 224 \\
\hline Traffic Accidents & 173 \\
\hline Other Accidents & 149 \\
\hline Others & 54 \\
\hline Injury & 45 \\
\hline Workplace Accident & 7 \\
\hline Suicide & 6 \\
\hline Fire injury & 3 \\
\hline
\end{tabular}

Table 2. Distribution of Calls Made by Syrians and Responded with Ambulance According to Reason of Call

\section{Discussion}

This study found differences compared with existing studies. Karakuş, et al analyzed the demographics and clinical features of Syrian refugees referred to Mustafa Kemal University Emergency Service in Hatay between June 2011 and July 2012 and found that the majority of the cases were male (88.8\%), and the most frequent complaint was a gunshot injury $(70.1 \%) .{ }^{10}$ However, in this study, female and male cases were nearly equal, and the leading reason for the call was medical. Regional differences may be the fundamental distinction affecting the results of the two studies. Although a surgical emergency was the leading cause for referral of emergency service near the Syrian border, medical causes were more common in cities like Ankara where Syrians resided. Similarly, male cases were higher in number in Hatay, whereas male and female cases were nearly equal in number in Ankara.

Considering the population that uses EMS services most commonly, it could be suggested that health planning should be done primarily for the younger population considering ongoing migration. Additionally, routine staff training should be revised considering the needs of this new patient profile.

The reason for an emergency call is valuable information for the ambulance medical team, as the team can review medical algorithms and prepare for the case. It is of utmost importance for dispatchers to effectively communicate with Syrian refugees on the phone. Therefore, staff, language, and cultural support should be 


\begin{tabular}{|l|c|c|c|}
\hline Time Intervals & Number (n) & Percent (\%) & $\mathbf{9 5 \%} \mathbf{C l}$ \\
\hline 00:00:00.001-06:00:00.000 & 337 & 14.8 & $13.3-16.5$ \\
\hline 06:00:00.001-12:00:00.000 & 416 & 18.3 & $16.6-20.1$ \\
\hline 12:00:00.001-18:00:00.000 & 770 & 33.9 & $31.6-36.4$ \\
\hline 18:00:00.001-00:00:00:000 & 747 & 32.9 & $31.8-36.6$ \\
\hline Total & $\mathbf{2 2 7 0}$ & $\mathbf{1 0 0 . 0}$ & - \\
\hline
\end{tabular}

Table 3. Distribution of Calls Made by Syrians and Responded with Ambulance According to Time Intervals of the Call

Note: Time interval is given as hh:mm:ss.fff.

provided to the Ankara Emergency Medical Centre, particularly for ambulance teams in the Altındağ region, as well as other cities with significant refugee populations. As stated in this study, most cases were referred for hospital emergency services. This means an additional workload and new patient profile for emergency services of hospitals. Language support is very important for hospital emergency service staff, as well as the EMS team.

Adequate service planning should be considered and support should be provided to ensure the well-being of the host society and refugees. The burden on the health care system should not be reflected on the host society, including local service providers.

\section{Limitations}

The study has some limitations. First is the retrospective nature of the study, which has inherent bias possible due to lack of pre-study definitions and control of data entry into the database source. Because the existing data from a database were used, it was not possible to customize the variables. The database has its own quality measures and the authors were not involved in the database process. However, database research is particularly appropriate for prehospital and disaster research because an emergency event or disaster cannot be controlled or easily predicted making prospective studies problematic. ${ }^{11}$ The study is limited to Syrians living in Ankara; it is difficult to generalize the findings to all contexts. Emergency health services are free to any one in Turkey, regardless of their status. But still, Syrians who are not registered and planned to flee to third countries might not use the EMS in order not to

References

1. El-Khatib Z, Scales D, Vearey J, Forsberg BC. Syrian refugees, between rocky crisis in Syria and hard inaccessibility to healthcare services in Lebanon and Jordan. Confl Health. 2013;7(1):18.

2. The UN Refugee Agency. 2015. http://data.unhcr.org/syrianrefugees/regional.php. Accessed December 20, 2015.

3. Ministry of Interior Directorate General Migration Management, Turkey. 2016. www.goc.gov.tr. Accessed March 21, 2016.

4. Nacar OA. Platform of health thought and medicine culture. 2016. http://www. sdplatform.com/Dergi/851/Suriyeli-siginmacilara-sunulan-saglik-hizmetleri.aspx. Accessed April 4, 2016.

5. Doocy S, Lyles E, Akhu-Zaheya L, Burton A, Weiss W. Health service utilization and access to medicines among Syrian refugee children in Jordan. Int J Health Plann Manage. 2016;31(1):97-112.

6. Ay M, Arcos González P, Castro Delgado R. The perceived barriers of access to health care among a group of non-camp Syrian refugees in Jordan. Int J Health Serv. 2016; 46(3):566-589. jeopardize their situation. Hence, such cases might be missing in the study. Despite these limitations, this is the first study to investigate prehospital EMS utilization by Syrians in Turkey.

\section{Conclusion}

Many Syrians have immigrated to Turkey since April 2011 due to the civil war in Syria. According to data obtained from the Ministry of Interior Directorate General Migration Management dated March 2016, the number of Syrian refugees in Turkey was $2,747,946$. Some of the Syrian refugees live in camps, whereas 2,475,134 live in metropolitan areas, such as Ankara. The number of EMS calls made by Syrian refugees rose from the beginning of 2011 to the first five months of 2015. The EMS system is used by Syrian refuges in all time periods, but usage after 18:00 was more common. The first five stations, which responded to nearly one-half $(42.0 \%)$ of all calls, are in the Altındağ region, which is densely populated by Syrian refugees. In Ankara, prehospital EMS was used mostly by Syrian refugees less than 18-years-old, which likely reflects the age distribution of Syrian refugees. Patients were mostly hospitalized in public hospitals.

\section{Acknowledgements}

The authors thank the Department of 112 Emergency Health Services of Ankara Provincial Health Directorate and its staff for their valuable support in this study.

7. World Vision. Advocacy report under pressure: the impact of the Syrian refugee crisis on host communities in Lebanon, 2013. http://www.worldvision.org/resources.nsf/main/ pressreports/\$file/Syria-Under-Pressure-Report_20130715.pdf. Accessed April 4, 2016.

8. Long BJ, Serrano LA, Cabanas JG, Bellolio MF. Opportunities for Emergency Medical Services (EMS) care of syncope. Prehosp Disaster Med. 2016;31(4):349-352.

9. Wein TH, Staub L, Feldberg R, et al. Activation of Emergency Medical Services for acute stroke in a non-urban population. Stroke. 2000;31(8):1925-1928.

10. Karakuş A, Yengil E, Akkücük S, Cevik C, Zeren C, Uruc V. The reflection of the Syrian civil war on the emergency department and assessment of hospital costs. Ulus Travma ve Acil Cerrahi Derg. 2013;19(5):429-433.

11. Stratton SJ. Using pre-existing databases for prehospital and disaster research. Prehosp Disaster Med. 2015;30(1):1-3. 